\title{
In situ XRD Study of Rapid Crystallization of Metallic Glasses Using Novel Experimental Setup for Flash-Annealing
}

\author{
J. BednarČÍK ${ }^{a, *}$, K. Kosiba ${ }^{b}$, S. PAuly ${ }^{b, c}$ And A. RothKirCH ${ }^{d}$ \\ ${ }^{a}$ P.J. Šafárik University, Institute of Physics, Park Angelinum 9, 04154 Košice, Slovakia \\ ${ }^{b}$ Institute for Complex Materials, IFW Dresden, Helmholtzstr. 20, 01069 Dresden, Germany \\ ${ }^{c}$ University of Applied Sciences, Würzburger Straße 45, 63743 Aschaffenburg, Germany \\ ${ }^{d}$ Deutches Elektronen-Synchrotron DESY, FS-EC, Notkestr. 85, 22603 Hamburg, Germany
}

\begin{abstract}
Fe-based metallic glasses (MGs) are known to exhibit excellent soft magnetic properties. A very effective way to further improve their magnetic properties is to incorporate nanocrystals into the glassy matrix. Usually it is done by conventional annealing in the vicinity of primary crystallization temperature. Direct current fast Joule heating (flash-annealing) represents an alternative way to introduce fine and randomly dispersed nanocrystals within MGs. Achieving high heating/cooling rates (well above $1000^{\circ} \mathrm{C} / \mathrm{s}$ ) by flash-annealing opens up new possibilities for study the fast kinetics. Phase composition and microstructure of a material can be tailored by proper heat-treatment in order to improve its overall performance. In this paper, we introduce a novel setup for studying rapid crystallization of metallic glasses using an in situ X-ray diffraction flash-annealing.
\end{abstract}

DOI: 10.12693/APhysPolA.137.852

PACS/topics: metallic glass, flash-annealing, crystallization, X-ray diffraction

\section{Introduction}

Nanocrystalline soft magnetic alloys are essential for variety of modern applications, and are commonly prepared by the devitrification of amorphous alloys obtained by rapid solidification [1]. Therefore, a phase transformation that has significant scientific interest is crystallization of metallic glasses. Under steady-state conditions, the heated glass transforms to the thermodynamically stable crystals through nucleation and growth [2]. However, the phase selection and the nucleation kinetics exhibit a pronounced heating-rate dependence when heated far from steady state [3]. Especially the early stages of crystallization are affected because as the nucleation rate decreases the glass is heated even faster [4]. The exact details of how the phase selection, the nucleation rate and crystal growth of different glass-forming alloys depend on the heating rate has not been investigated to date. Recently, there were proposed several concepts of fast-heating such as inductive [4], microwave [5] and direct-current Joule [6] heating. Our contribution to this field is introduction of a novel setup for studying rapid crystallization of metallic glasses using an in situ $\mathrm{X}$-ray diffraction flash-annealing.

\section{Experimental}

Amorphous ribbons with nominal composition of $\mathrm{Fe}_{73.5} \mathrm{Cu}_{1} \mathrm{Nb}_{3} \mathrm{Si}_{15.5} \mathrm{~B}_{7}$ (at.\%) were prepared by a single- roller melt-spinning technique. In situ X-ray diffraction (XRD) experiments were performed in transmission mode at the P02.1 beamline of the PETRA III electron storage ring (Hamburg, Germany). The synchrotron radiation wavelength was $\lambda=0.02075 \mathrm{~nm}$, and the beam size was set to $0.5 \times 0.5 \mathrm{~mm}^{2}$. Two-dimensional detector Dectris PILATUS 3 CdTe (pixel size $172 \times 172 \mu \mathrm{m}^{2}$ ) positioned $115 \mathrm{~mm}$ downstream from the heating cell was used to collect scattered photons. Flash-annealing experiments were set according to scheme shown in Fig. 1. Each experiment starts with a piece $(10 \mathrm{~mm}$ long, $2 \mathrm{~mm}$ wide and $20 \mu \mathrm{m}$ thick) of amorphous ribbon fixed between two electrodes of the custom made heating cell. Both electrodes of the heating cell are connected to the four quadrant current amplifier Toellner TOE 7610-20, which amplifies an incoming voltage pulse generated by an arbitrary wave-form generator Hameg HMF 2550. The custom made heating cell has windows on both sides allowing XRD in transmission mode. Flashannealing was realized by generating a burst of 20 rectangular pulses with repetition rate of $0.1 \mathrm{~s}$ (frequency $1 \mathrm{~Hz}$ ) and pulse width $40 \mathrm{~ms}$. Such a train of rectangular pulses is further amplified by a current amplifier, producing an electrical current with desired time structure. Amplitude, width and repetition rate of a current pulse determine amount of heat generated within the specimen. Two-dimensional (2D) XRD patterns were recorded with temporal resolution of $0.1 \mathrm{~s}$ (acquisition rate of $10 \mathrm{~Hz}$ ). Azimuthal integration of 2D XRD patterns was done using a pyFAI program [7]. 


\section{Results and discussion}

Several offline tests without X-ray beam were done in order to find out a proper set of rectangular pulse parameters such as amplitude and width. One should keep in mind that keeping the sample's geometry (length and cross-section) unchanged ensures same value of resistance, and thus makes process of parameters optimization more reliable. Offline tests confirmed that rectangular pulses with amplitude and width of $1.5 \mathrm{~A}$ and $30-60 \mathrm{~ms}$, respectively, are sufficient to crystallize $\mathrm{Fe}_{73.5} \mathrm{Cu}_{1} \mathrm{Nb}_{3} \mathrm{Si}_{15.5} \mathrm{~B}_{7}$ alloy. In turn, higher amplitudes and longer pulses cause alloy melting.

Figure 2 shows series of XRD patterns recorded during in situ flash-annealing performed with a burst of 20 rectangular pulses having the amplitude and width of 1.5 and $40 \mu \mathrm{m}$, respectively. XRD patterns acquired before arrival of the first pulse confirm fully amorphous nature of as-cast alloy. The sample immediately crystallizes when the first current pulse arrives. As a result set of sharp and intense Bragg peaks corresponding to cubic $\mathrm{Fe}_{3} \mathrm{Si}$ phase is observed. In addition traces of boride phase such as $\mathrm{Fe}_{23} \mathrm{~B}_{6}$ are visible. Previous studies show that the onset temperatures of primary and secondary crystallization determined by differential scanning calorimetry at heating rate of $10 \mathrm{~K} / \mathrm{min}$ are $T_{x 1}=508^{\circ} \mathrm{C}$ and $T_{x 2}=694^{\circ} \mathrm{C}$, respectively [8]. Formation of boride phases usually takes place during secondary crystallization, so one may conclude that the first current pulse caused temperature rise to at least $700^{\circ} \mathrm{C}$. As the first pulse fades away sample's temperature decreases due to dissipation of deposited heat. Such behavior is reflected by increasing intensity of Bragg peaks and shifting their positions towards larger magnitudes of scattering vector $q$. Consecutive current pulses do not influence phase composition which remains unchanged after finishing of all 20 pulses. One can observe only

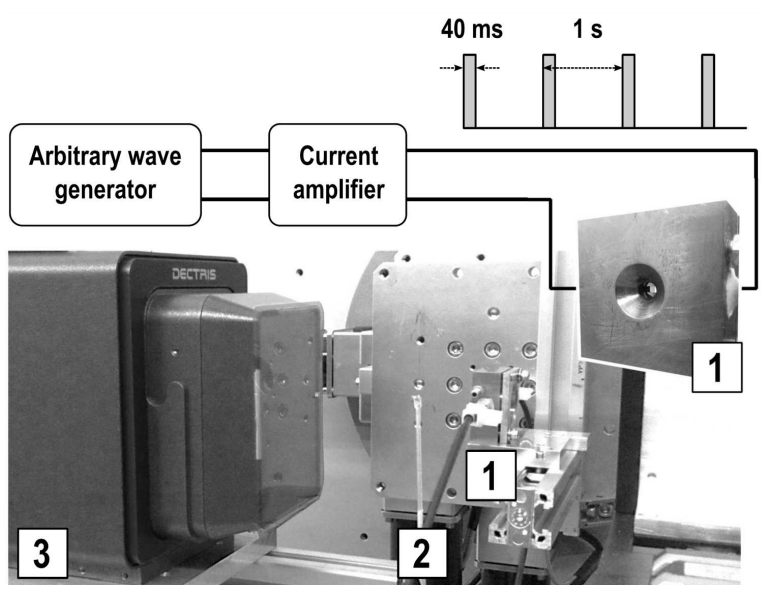

Fig. 1. Setup for flash-annealing experiments at the P02.1 beamline consisting of an arbitrary wave generator, current amplifier, custom made heating cell (1), beamstop (2) and two-dimensional X-ray detector (3). At the top a typical current pulse train is depicted.

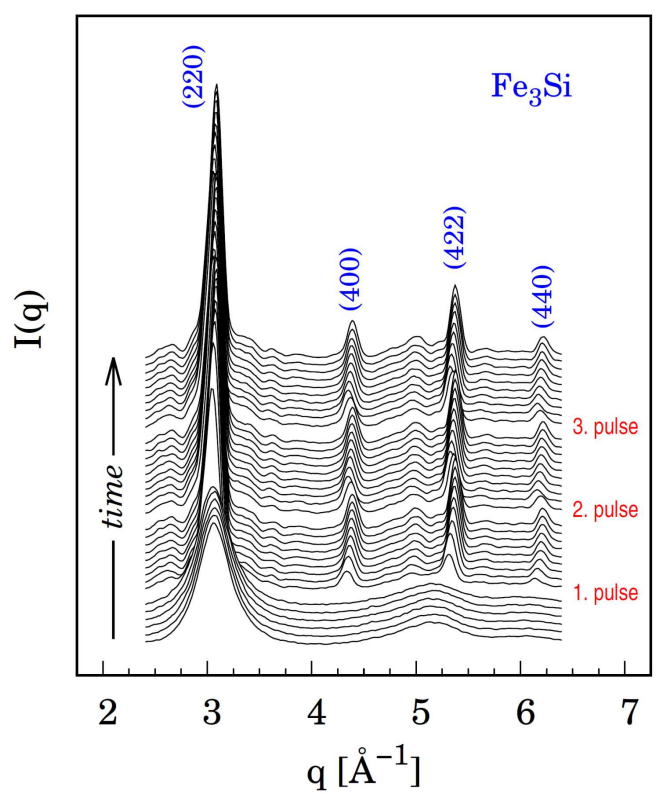

Fig. 2. Series of XRD patterns acquired in transmission mode with acquisition rate of $10 \mathrm{~Hz}$. Patterns are vertically offset and grouped based on arriving pulses for better clarity. The strongest reflections of the cubic $\mathrm{Fe}_{3} \mathrm{Si}$ phase are labeled with Miller indices.

temperature effects associated with the variations of peak intensity and position. In order to characterize these changes quantitatively, profile of the (422) Bragg peak (corresponding to the $\mathrm{Fe}_{3} \mathrm{Si}$ phase) was fitted using a Gaussian function. In case of a cubic phase the lattice constant $a$ can be calculated from the position $2 \theta_{h k l}$ of the Bragg peak with Miller indices $h$, $k$ and $l$. It is expressed with the following equation $a=\lambda \sqrt{h^{2}+k^{2}+l^{2}} /\left(2 \sin \left(\theta_{h k l}\right)\right)$, in which $\lambda$ is the wavelength.

Figure 3 shows time dependence of the lattice constant $a$, which perfectly matches with the time structure of rectangular pulses. It can be seen that sample gets suddenly heated with arrival of a pulse, which is manifested by thermal expansion of the crystal lattice. Before next pulse arrives, specimen spontaneously cools down as seen by an exponential decay of the lattice parameter $a$. On the other hand the full-width at halfmaximum (FWHM), of the (422) Bragg reflection does not change significantly with arriving pulses. It suggests rather that a stable microstructure was already established by the first current pulse, while consecutive pulses result in cyclic thermal expansion of a crystal lattice.

After arrival of the last current pulse the sample cools down, and can reach a thermal equilibrium at an ambient conditions (see Fig. 4). Time behavior of the lattice constant $a(t)$ was fitted using a function $a(t)=a_{\mathrm{ref}}+a_{0} \mathrm{e}^{-k\left(t-t_{0}\right)}$, where $a_{0}$ is the offset to the lattice constant at the time $t_{0}, k$ is the rate parameter, and $a_{\text {ref }}$ is the value of the lattice constant in thermal equilibrium (when $t \rightarrow \infty$ ). Obtained values for $a_{\text {ref }}$ and $k$ are 5.6992(2) $\AA$ and 2.33(5) s ${ }^{-1}$, respectively. 


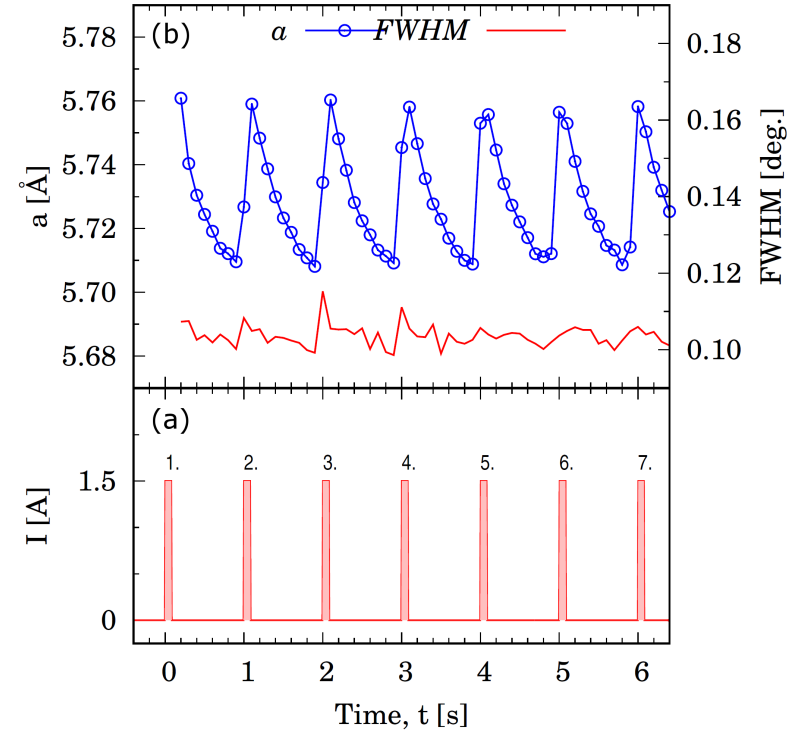

Fig. 3. (a) Time structure of rectangular current pulses with amplitude of $1.5 \mathrm{~A}$ and duration of $40 \mathrm{~ms}$. Only the first seven pulses (out of 20) are shown. (b) Temporal evolution of the lattice constant $a$ derived from the position of the (422) Bragg reflection and time behaviour of its FWHM.

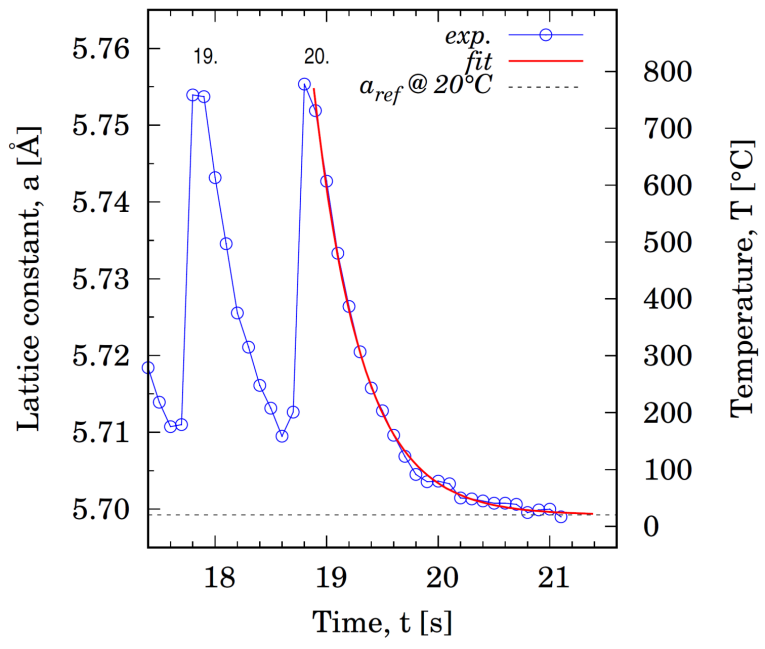

Fig. 4. Time dependence of the lattice constant $a$ after the last, i.e. 20th, current pulse. The solid (red) line represents a fit with an exponential decay function to the experimental data (o).

Assuming an average value for the coefficient of thermal expansion of $\mathrm{Fe}_{3} \mathrm{Si}$ phase between 20 and $700^{\circ} \mathrm{C}$ is $\alpha=1.3 \times 10^{-5} \mathrm{~K}^{-1}[9]$, one can relate the values of the lattice constant $a$ with temperature using an equation $a(T)=a_{\text {ref }}\left[1+\alpha\left(T-T_{\text {ref }}\right)\right]$, where $a_{\text {ref }}$ is the lattice constant at the reference temperature $T_{\text {ref }}=$ $20^{\circ} \mathrm{C}$ (see Fig. 4). The mean value of the heating rate estimated from slopes of the rising edges of all 20 pulses is approximately $4500^{\circ} \mathrm{C} / \mathrm{s}$.

\section{Conclusions}

In this work a novel setup for flash-annealing experiments is presented, which was implemented and successfully tested at the P02.1 beamline. Its potential use was demonstrated by studying rapid crystallization of soft magnetic Fe-based metallic glass. It was shown that a single rectangular current pulse with an amplitude and duration of $1.5 \AA$ and $40 \mathrm{~ms}$, respectively, crystallizes amorphous $\mathrm{Fe}_{73.5} \mathrm{Cu}_{1} \mathrm{Nb}_{3} \mathrm{Si}_{15.5} \mathrm{~B}_{7}$ ribbon by heating it up to $800^{\circ} \mathrm{C}$ with an average heating rate of $4500{ }^{\circ} \mathrm{C} / \mathrm{s}$. Phase composition of crystallized material consists of major $\mathrm{Fe}_{3} \mathrm{Si}$ phase and small traces of $\mathrm{Fe}_{23} \mathrm{~B}_{6}$ phase. Thermal expansion behaviour of $\mathrm{Fe}_{3} \mathrm{Si}$ phase was used to determine temperature at various stages of flash-annealing. Further works are planned to test higher temporal resolution of data acquisition and direct temperature measurement with a pyrometer.

\section{Acknowledgments}

This study was partially supported by the grant VEGA 1/0036/16. K.K. thanks financial support through the German Science Foundation (DFG) under grant No. KO 5771/1-1. Parts of this research were carried out at the light source PETRA III (beamline P02.1) at DESY, a member of the Helmholtz Association (HGF). This work was supported by the project ITMS: 313011T544, co-funded by the European Regional Development Fund (ERDF).

\section{References}

[1] G. Herzer, J. Magn. Magn. Mater. 157-158, 133 (1996).

[2] K.F. Kelton, Solid State Phys. 45, 75 (1991).

[3] V.A. Shneidman, J. Chem. Phys. 102, 1791 (1995).

[4] K. Kosiba, S. Pauly, Sci. Rep. 7, 2151 (2017).

[5] R. Nicula, M. Stir, K. Ishizaki, J.M. Catala-Civera, S. Vaucher, Scr. Mater. 60, 120 (2009).

[6] N. Mitrović, S. Kane, S. Roth, A. Kalezić-Glišović, C. Mickel, J. Eckert, J. Min. Metall. B 48, 319 (2012).

[7] J. Kieffer, D. Karkoulis, J. Phys. Conf. Ser. 452, 202012 (2013).

[8] G. Herzer, V. Budinsky, C. Polak, Phys. Status Solidi B 248, 2382 (2011).

[9] M.C.M. Farquhar, H. Lipson, A.R. Weill, J. Iron Steel Inst. 152, 457 (1945). 